\title{
Emergency Evacuation Bottleneck of Pas- sengers in the Seaport Station
}

\author{
Guoqing Sun
}

China Waterborne Transport Research Institute, Beijing 100088, China

\begin{abstract}
Seaport passenger passengers evacuation problems for optimizing the research design and strengthen seaport passenger passengers emergency effective management measures, by improving port passenger safety of passengers emergency significance. In this paper the research on both domestic and foreign passenger evacuation was summarized. Discuss the passenger evacuation marks port passenger press release and emergency evacuation marks for passenger evacuation play a directive role, and effectively save evacuation time.
\end{abstract}

Keywords: Emergency Evacuation; Seaport Passengers; Passengers safety; Evacuation time; Evacuation bottleneck

\section{Introduction}

Nowadays, Passengers emergency evacuation has become a severe problem in many public Station of cities, because the public station capacity can not satisfy the ever growing traffic demand. We know that the public station capacity is greatly determined by the station bottlenecks. Thus the problem of passengers emergency evacuation can be alleviated greatly by improving the capacity of station bottlenecks.

Due to merging or diverging behaviors, lane changes are very common at station bottleneck. This makes the passengers emergency evacuation in disorder state and reduces the capacity. In peak hours, very serious congestion always happens at station bottleneck. The dynamics of traffic flow at bottlenecks should be firstly understood then some control measures can be made to keep the passengers emergency evacuation in high flow states.

Establishing more realistic simulation models is very helpful for understanding the dynamics of passengers emergency evacuation flow. Many theoretical models have been proposed to explore the evolution mechanism of passengers emergency evacuation flow ${ }^{[1]}$.Among those models, cellular automata (CA) model has become an excellent tool for modelling passengers emergency evacuation flow. In 1992, Nagel and Schreckenberg proposed the well-known Nagel-Schreckenberg (NaSch) model [2]. Although it is very simple, complex passengers emergency evacuation phenomena, such as stop-and-go traffic and fundamental diagram, can be reproduced.

Based on the NaSch model, many works have been done to investigate the traffic dynamics around bottlenecks [3-4]. Traffic networks are very complex systems where elaborated topologies are combined with large number of passengers running on the network. Predicting traffic behavior is very important for planning and operation purposes. In the last years, computer simulations as means for evaluating control and management strategies in passengers emergency evacuation systems have gained considerable 
importance because of the possibility of taking into account the dynamical aspects of passengers emergency evacuation.

In principle, traffic simulation models can roughly be divided into macroscopic and microscopic ones. While macroscopic models examine the dependencies between traffic flow, traffic volume, and average velocities; microscopic models investigate the movements of individual passengers emergency evacuation. In general, passengers flow models should keep the description of the relevant aspects of the flow dynamics as simply as possible by keeping track of the essential. In this spirit, Cellular Automata (CA) models for passengers flow were developed. Its main advantage is an efficient and fast performance when used in computer simulations, due to their rather low accuracy on a microscopic scale. These CA models for passengers flow are discrete in nature, in the sense that time advances with discrete steps, space is coarse-grained and properties of the CA can have only a finite, countable number of states allowing for high-speed simulations, especially when they are performed on a platform for parallel computation $^{[1,2,3]}$.

\section{Passengers Emergency Evacuation Model in Seaport}

\subsection{Rules and Law}

The model presented here is a probabilistic cellular automaton. It consists of $\mathrm{N}$ passengers moving in one direction on a one-dimensional lattice of $\mathrm{L}$ cells arranged in a ring topology. The number of passengers is fixed. Each cell can either be empty, or occupied with a single passenger that spans one or more consecutive cells. The velocity of a passenger is constrained to an integer in the range $\left\{0, \ldots, V_{\max }\right\}$. In this paper, passengers are allowed to occupy more than one cell. The speed limit, $\mathrm{V}_{\max }$ can be different depending on the kind of passenger under consideration: trucks, cars, etc. For simplicity, in this paper only one type of passenger is considered and therefore the same maximum velocity will be used for all passengers. The integer velocity, that corresponds to one of the passenger states in this CA, is related with the number of cells that a passenger advances in one time step3. The other state, position, is related with the cell or cells that each passenger is occupying Expressions in equations (1) to (3) represent the safe distances the n-th passenger must have with respect to its preceding passenger if it is going to accelerate, (1), keep its velocity, (2), or decelerate, (3) in the current time step. The basis to calculate these safe distances is to assume that the worst possible scenario after any of the these three basic maneuvers is performed corresponds to the passenger in front applying full stops $[4,5,6]$

There are two groups of terms in the right-hand side (RHS) of each one of the expressions given in equations (1) to (3). The first two terms represent the traveled distance by the passenger $\mathrm{n}$ assuming that in the next time-step it finds that the passenger $\mathrm{n}+1$, in front of it, has slammed the stops; forcing passenger $n$ to also hit the stops.

The last two terms in the RHS of the expressions given in (1) to (3) are the traveled distance by passenger $n+1$ if at the current time-step it slams the stops. To determine state transitions the following set of rules, which are applied simultaneously to all passengers, is defined:

$\mathrm{R}_{0}$ : Calculate $\operatorname{ddecc}(\mathrm{t})$, $\operatorname{dacc}(\mathrm{t})$, and dkeep(t)

$\mathrm{R}_{1}$ : Acceleration.

If $\operatorname{dn}(\mathrm{t}) \geqslant \operatorname{daccn}(\mathrm{t})$, the velocity of the car $\mathrm{n}$ is increased by one, i.e., 
$\mathrm{Vn}(\mathrm{t}+1) \rightarrow \min (\mathrm{Vn}(\mathrm{t})+1, \mathrm{Vmax})$

$\mathrm{R}_{2 \mathrm{a}}$ : Cruising.

If $\operatorname{daccn}(\mathrm{t})>\operatorname{dn}(\mathrm{t}) \geqslant$ dkeepn $(\mathrm{t})$, velocity of passenger $n$ is kept equal with probability1-R, i.e., $\mathrm{Vn}(\mathrm{t}+1) \rightarrow \mathrm{Vn}(\mathrm{t})$ with probability 1-R.

$\mathrm{R}_{2 \mathrm{~b}}$ : Random stopping.

If $\operatorname{daccn}(\mathrm{t})>\operatorname{dn}(\mathrm{t}) \geqslant$ dkeepn $(\mathrm{t})$ and $\mathrm{Vn}(\mathrm{t})>0$, velocity of passenger $\mathrm{n}$ is reduced by one with probability $\mathrm{R}$ :

$\mathrm{Vn}(\mathrm{t}+1) \rightarrow \max (\mathrm{Vn}(\mathrm{t})-1,0)$ with probability R.

$\mathrm{R}_{3}$ : Stopping.

If dkeepn $(\mathrm{t})>\operatorname{dn}(\mathrm{t}) \geqslant \operatorname{ddeccn}(\mathrm{t})$ and $\mathrm{vn}(\mathrm{t})>0$, velocity of passenger $\mathrm{n}$ is reduced by one:

$$
\mathrm{Vn}(\mathrm{t}) \rightarrow \max (\mathrm{Vn}(\mathrm{t})-1,0)
$$

$\mathrm{R}_{4}$ : Emergency stopping.

If $\mathrm{Vn}(\mathrm{t})>0$ and $\operatorname{dn}(\mathrm{t})<\operatorname{ddeccn}(\mathrm{t})$, velocity of passenger $n$ is reduced by $M$, provided it does not go below zero:

$$
\mathrm{Vn}(\mathrm{t}+1) \rightarrow \max (\mathrm{Vn}-\mathrm{M}, 0)
$$

$\mathrm{R}_{5}$ : Passenger movement.

Each passenger is moved forward according to its new velocity determined in rules 1-4:

$$
\mathrm{Xn}(\mathrm{t}+1) \rightarrow \mathrm{Xn}(\mathrm{t})+\mathrm{Vn}(\mathrm{t}+1)
$$

Rules $R_{1}$ to $R_{4}$ are designed to update velocity of passengers; rule $R_{5}$ updates position. According to this, state updating is divided into two stages, first velocity, second position. The rationale behind rules $R_{1}$ to $R_{4}$ is as follows.

$\mathrm{R}_{1}$ : This rule postulates that all the passengers strive to reach the maximum velocity whenever possible. This is in agreement with other velocity policies, as it is the case with the greedy policy in ${ }^{[3]}$.

$\mathrm{R}_{2 \mathrm{a}}$ : This rule reflects the fact that passengers will try to keep their velocity if they perceive the distance with the passenger in front as safe.

$\mathrm{R}_{2 \mathrm{~b}}$ : This rules is introduced to model traffic disturbances that cause passengers to reduce their speed for no apparent reason. These can happen, for example, due to incidents along the station that distract passengers. This random stopping contributes to creation of traffic jams.

$R_{3}$ : This rules requires the passenger to apply moderate stopping when the spacing that separates his/her passenger to the passenger in front is becoming small.

$\mathrm{R}_{4}$ : This rule stresses the approach taken in this paper: the most important passengers' decisions are related to safety. Thus, when according to its speed and the speed of the passenger in front, the passenger perceives an unsafe spacing, he/she will slam the stops. When conditions for this rule are met, passenger is in an unsafe situation that could lead to a collision if the passenger in front slam the stops. For this reason the passenger of the passenger will slam the stops. If initial distributions of relative distances to the velocity are selected to satisfy at least ddecc, then passengers will never have a relative distance such that this rule is activated. This rule was introduced to proof this fact and to allow also perturbations in the other rules trying to investigate their effect.

There are several modification to the Nash model. First and most important, it should be noted that velocity setting rules depend not only on the relative velocity of neighbor passengers, they now take into account their relative distance. This modification was included to incorporate normal passengers behavior that base their driving decisions on both relative velocity and relative distance. It should be noted that safe distances given in equations (1) to (3) grow faster than linear with relative velocity of passengers.

This is in accordance with normal passengers spacing policies. The values that are used here are much closer to those reported for normal driving. Note that the order for applying rules $R_{1}$ to $R_{4}$ is not relevant as conditions for each one form disjoint sets. Other relevant modification to the Nash-model is the 
change in the application of the deceleration and randomization rules. In the Nash-model, randomization is applied after deceleration, while in the model here proposed randomization is applied only to passengers that are in cruising conditions and do not require to stop.

The only probabilistic behavior is included in rule $R_{2 b}$. It should be noted, however, that the value of the probability of random stopping will be smaller than that reported in. However, additional rules may be incorporated to capture more complex situations. The parameters of the model are the following: number of cells L, maximum number of passengers Nmax, number of passengers driving $\mathrm{N}$, limit speed $\mathrm{V}_{\max }$, passenger length $\mathrm{s}$, number of time steps to achieve maximum stopping $M$ and the random stopping probability in cruising $\mathrm{R}$.

\subsection{Simulation}

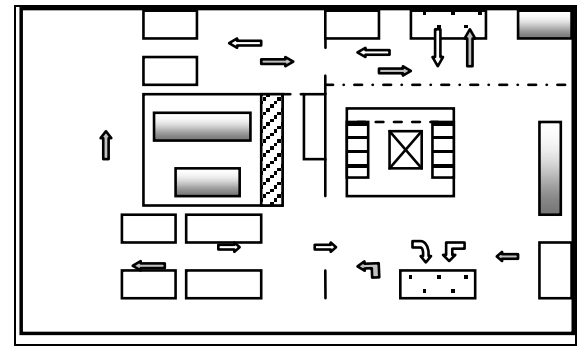

Fig. 1: The layout diagram of the Tianjin Seaport Station.

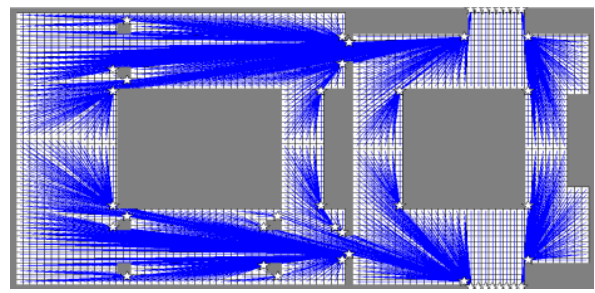

Fig. 2: The bottleneck of passenger evacuation in Tianjin Seaport Station.
In this paper a modification of the Nash model to better capture passenger reactions to traffic that are intended to preserve safety on the station was introduced and investigated. As a result, three distances that represent the safety distance that a passenger must have with respect to preceding passenger if it is going to decelerate, keep its velocity or accelerate, were included in the new model. The addition of these distances allowed to determine the most appropriate action for a passenger to undertake based on the distance from the passenger ahead of it and the velocities of the two neighbor passengers.

\subsection{Acknowledgments}

This paper is the product of an effort undertaken by the projects of Chinese Safety Committee of passenger public. The writer is grateful for the technical assistance provided by all committee members.

\section{References}

[1] K. Nagel and M. Schreckenberg. A Cellular automaton model for freeway traffic. Journal Physique I, 2:2221-2229, 1992.

[2] B. Kerner, S. L. Klenov, D. E. Wolf, Cellular automata approach to threephase traffic theory. Journal of Physics A, 35: 9971-10013, 2002.

[3] G. Diedrich, L. Santen, A. Schadschneider, and J. Zittartz, Effects of on- and off-ramps in cellular automata models for traffic flow. International Journal of Modern Physics $C, 11: 335-345,2000$. 